\title{
Soluções de condicionamento em pós-colheita de inflorescências de antúrio
}

\author{
Edson Shigueaki Nomura ${ }^{1}$ Eduardo Jun Fuzitani ${ }^{2}$, Erval Rafael Damatto Junior ${ }^{3}$
}

\section{RESUMO}

Objetivou-se, com este trabalho, avaliar o uso de soluções de condicionamento pós-colheita, em diferentes cultivares de antúrio. O experimento foi conduzido sob delineamento em blocos ao acaso, em esquema fatorial 3 x 3 (cultivar x solução de condicionamento), sendo que os cultivares testados foram: 'IAC Juréia', 'IAC Eidibel' e 'Apalai' e as soluções de condicionamento foram: água, água+sacarose $(5,0 \%)$ e água+ácido cítrico $\left(200 \mathrm{mg} \mathrm{L}^{-1}\right)$. O condicionamento foi realizado por meio da imersão das inflorescências em 0,5 L de solução, por 24 horas, e posteriormente, sua manutenção em igual volume de água destilada, renovada a cada quatro dias. A cada renovação da água, avaliaram-se a massa da matéria fresca, a durabilidade comercial e a longevidade total, segundo o critério de notas. Os resultados foram submetidos à análise de variância e ao teste F, e, quando significativos, as médias foram comparadas pelo teste de Tukey $(p<0,05)$. Com os dados obtidos, conclui-se que não houve diferenças entre as soluções de condicionamento para os diferentes cultivares, sendo que o 'IAC Juréia' apresentou maior durabilidade comercial (21 dias) e longevidade total (48 dias), independentemente da solução de condicionamento.

Palavras-chave: Anthurium andraeanum, sacarose, ácido cítrico.

\section{ABSTRACT}

\section{Anthurium flowers post-harvest conditioning}

Aiming to obtain flowers with greater longevity, this study aimed to evaluate the use of post-harvest conditioning solutions in different cultivars of anthurium. The experiment was carried out in randomized block design in a factorial scheme 3 x 3 (cultivar x conditioning solution). The tested cultivars were: Juréia IAC, IAC Eidibel and Apalai. The conditioning solutions were: water, water + sucrose $(5.0 \%)$ and water + citric acid $\left(200 \mathrm{mg} \mathrm{L}^{-1}\right)$. Conditioning was performed by immersing the flowers in $0.5 \mathrm{~L}$ of conditioning solution for 24 hours and subsequently maintained with an equal volume of distilled water renewed every four days. At each water renewal, was evaluated the fresh weight, durability and longevity adopting notes. The results were submitted to ANOVA and F test, and means were compared by Tukey test $(p<0,05)$ when significant. With the obtained data it can be concluded that there was no difference between the conditioning solutions for different cultivars, and 'IAC Juréia' showed greater durability shopping (21 days) and total longevity (48 days), regardless of the conditioning solution.

Key words: Anthurium andraeanum, sucrose, citric acid.

\footnotetext{
Recebido para publicação em 15/06/2012 e aprovado em 08/07/2013.

${ }^{1}$ Engenheiro-Agrônomo, Mestre. Agência Paulista de Tecnologia dos Agronegócios (APTA), Pólo Regional Vale do Ribeira, Rodovia BR 116, Km 460, Caixa Postal 122, 11900000, Registro, São Paulo, Brasil. edsonnomura@apta.sp.gov.br (autor para correspondência).

${ }^{2}$ Engenheiro-Agrônomo. Agência Paulista de Tecnologia dos Agronegócios (APTA), Pólo Regional do Vale do Ribeira, Rodovia BR 116, Km 460, Caixa Postal 122, 11900-000, Registro, São Paulo, Brasil. edufuzitani@apta.sp.gov.br

${ }^{3}$ Engenheiro-Agrônomo, Doutor. Agência Paulista de Tecnologia dos Agronegócios (APTA), Pólo Regional do Vale do Ribeira, Rodovia BR 116, Km 460, Caixa Postal 122, 11900000, Registro, São Paulo, Brasil. erval@ apta.sp.gov.br
} 


\section{INTRODUÇÃO}

O antúrio (Anthurium andraeanum Lind.) é uma espécie da família Araceae, ordem Alismatales, classe Liliopsida, caracterizada por espécies com inflorescências em espádice e protegida por espata (Castro et al., 2012). Essa família abrange 106 gêneros e cerca de 2823 espécies (Govaerts \& Frodin, 2002). Na realidade, a flor comercial de antúrio é uma inflorescência, composta de um pedúnculo, uma bráctea colorida, chamada de espata, e uma pequena "espiga" chamada espádice, onde se encontram agregadas dezenas de pequenas flores seguindo um delineamento espiralado (Castro et al., 2012). Tem grande valor ornamental, sendo usado como planta de vaso, para jardins e interiores com pouca incidência de sol. Dada sua alta durabilidade pós-colheita e conformação típica, segundo Castro et al. (2004), o antúrio é largamente utilizado na floricultura e no paisagismo, sendo, dentre as plantas de clima tropical, a segunda mais consumida no mundo, superada apenas pelas orquídeas. Segundo Paull \& Goo (1985), o tamanho, a forma e a coloração da espata determinam o valor comercial das inflorescências; além disso, o tamanho e a orientação da espádice são critérios adicionais de qualidade.

A região do Vale do Ribeira possui cerca de 812,2 ha, em 242 unidades de produção agrícola (UPAs), com floricultura para vaso e viveiros de flores e plantas ornamentais (LUPA, 2009) e, dentre elas, encontra-se o cultivo comercial do antúrio. Estima-se a existência de cerca de 1,7 milhões de plantas de antúrio, em cultivo, no Vale do Ribeira (Tombolato et al., 2002). Os produtores dessa região tradicionalmente cultivam o antúrio sob viveiro coberto com malha de coloração preta e sombreamento, variando de 50 a $80 \%$, dependendo da época do ano.

O programa de melhoramento da Agência Paulista de Tecnologia dos Agronegócios - Instituto Agronômico, Campinas, SP (APTA/IAC), selecionou, nas últimas décadas, os primeiros cultivares brasileiros de antúrio, que vêm sendo micropropagados e fornecidos aos produtores de todo o país. Dentre eles destacam-se: 'IAC Astral', 'IAC Cananéia', 'IAC Eidibel', 'IAC Iguape', 'IAC Isla', 'IAC Luau', 'IAC Juréia', 'IAC Ômega', 'Júpiter', 'Juquiá’, 'Netuno' e 'Rubi' (Castro et al., 2012).

Atualmente, o 'IAC Eidibel' é o principal cultivar explorado comercialmente, para corte, no Brasil, estimando-se cerca de 500 mil plantas em cultivo (Tombolato et al., 2004). Caracteriza-se por apresentar planta vigorosa de porte médio, altamente produtiva e de crescimento rápido, espata cordiforme de tamanho médio, textura grossa, com boa enervação, de coloração vermelho forte e espádice branca, suavemente perfumada, sendo uma flor de corte de longa durabilidade pós-colheita (Tombolato et al., 2004).

Apesar da evolução na produção e comercialização do antúrio, grande parte do produto colhido é perdida durante o transporte, por causa dos danos causados pelo manuseio e das embalagens inadequadas e, principalmente, do armazenamento ineficiente (Leme, 2008). Os principais fatores que causam a perda precoce de flores cortadas são a desidratação, os baixos teores de açúcares, a presença de micro-organismos na água, as características do vaso, a temperatura ambiente, a ação do etileno e o estádio de desenvolvimento da flor no momento do corte (Kämpf, 1984). A adoção de medidas de controle dessas perdas faz-se indispensável, principalmente para a manutenção da qualidade de flores cortadas destinadas à exportação (Castro et al., 1983).

A utilização de soluções preservativas e, ou, de compostos químicos para manter a qualidade e prolongar a vida das flores cortadas tem crescido bastante, sendo ação rotineira nos principais países produtores de flores. Entre os ingredientes utilizados para a conservação de flores de corte incluem-se a água, os açúcares, íons minerais, os germicidas, os inibidores de etileno, os reguladores de crescimento e os antioxidantes (Castro, et al., 1983). Normalmente, são utilizados quatro tipos de soluções para conservação pós-colheita de flores de corte, que podem ser classificadas, de acordo com o objetivo de uso, como soluções de condicionamento, de pulsing, de indução à abertura floral e de manutenção (Castro, 1993).

O uso de solução de condicionamento, antes ou após o armazenamento, pode ser definido como o tratamento utilizado nas primeiras 24 horas após a colheita. É considerado um tratamento rápido de pré-transporte ou armazenamento, que afeta a fase final da vida das flores, prolongando-a, mesmo após a transferência para a água ou solução de manutenção (Halevy \& Mayak, 1981).

A utilização de sacarose na solução de condicionamento é comum e a concentração pode variar de 2 a $20 \%$ (Dias-Tagliacozzo \& Castro, 2002). O efeito da solução de sacarose, tanto na forma de condicionamento, como na forma de solução em vaso, pode variar consideravelmente entre as espécies. Em algumas espécies, a sacarose tem sido eficiente no prolongamento da vida pós-colheita e na promoção de abertura de botões imaturos, propiciando colheita antecipada e maior vida pós-colheita da flor cortada (Van Doorn \& Reid, 1992). Além da sacarose, ácidos orgânicos também podem ser utilizados na solução de condicionamento, com o objetivo de reduzir o $\mathrm{pH}$ das soluções, pois pH ácido proporciona aumento da durabilidade de flores de corte, pela redução da ação dos microorganismos (Lima \& Ferraz, 2008).

Em virtude da carência de informações sobre a póscolheita de Anthurium andraeanum e visando à obtenção de flores com maior longevidade, este trabalho objetivou avaliar o uso de soluções de condicionamento em pós-colheita, em diferentes cultivares de antúrio, da APTA/IAC. 


\section{MATERIAL E MÉTODOS}

O experimento foi realizado no laboratório da Agência Paulista de Tecnologia dos Agronegócios (APTA) Regional Vale do Ribeira, município de Pariquera-Açú, SP. O clima da região é classificado, segundo Köppen, como Af: tropical chuvoso, sem estação seca, com precipitação média do mês mais seco superior a 60 mm. Dados de uma série de dez anos (2001 - 2011), registrados na estação meteorológica da APTA Pólo Regional do Vale do Ribeira, mostram que a média anual da temperatura máxima e mínina foram de $26,7^{\circ} \mathrm{C}$ e $17,1^{\circ} \mathrm{C}$, respectivamente, e a pluviosidade média foi de $1.715,6 \mathrm{~mm}$ anuais.

Utilizou-se delineamento experimental em blocos ao acaso em esquema fatorial 3 x 3 (cultivar x solução de condicionamento), com quatro repetições e três hastes florais por parcela, empregando-se os cultivares 'IAC Juréia', 'IAC Eidibel' e 'Apalai', e as soluções de condicionamento foram água (testemunha), água + sacarose $(5,0 \%)$ e água + ácido cítrico $\left(200 \mathrm{mg} \mathrm{L}^{-1}\right)$. As inflorescências de antúrio foram provenientes de uma área de produção comercial (Sítio Oriente), localizada nas proximidades da APTA - Pólo Regional do Vale do Ribeira.

A maturidade da inflorescência do antúrio foi estimada pelo grau de mudança de cor da espádice, sendo as inflorescências deste experimento colhidas quando metade a $3 / 4$ das flores verdadeiras estavam abertas (Lopes $\&$ Mantovani, 1980). Depois de colhidos, o comprimento das hastes florais dos diferentes cultivares foi padronizado $(50 \mathrm{~cm})$, realizando-se corte, na porção basal, em forma de bisel, para aumentar a superfície de absorção de solução pelas hastes florais e para que essa superfície não ficasse totalmente em contato com o fundo do recipiente, o que poderia prejudicar a absorção.

O condicionamento foi realizado por meio da imersão da base das hastes florais em 0,5 L de solução, por 24 horas. Posteriormente, as hastes foram mantidas em igual volume de água destilada, renovada a cada quatro dias. Durante o experimento, a temperatura e umidade relativa máxima e mínima foram: 25,6 e $21,3{ }^{\circ} \mathrm{C}$ e 76,5 e $57,6 \%$, respectivamente.

A cada renovação da água destilada, as hastes florais foram pesadas até o vigésimo dia após o período de condicionamento (24 horas), para avaliar o ganho ou perda de massa. Durante o processo de senescência, efetuaram-se avaliações da durabilidade comercial e da longevidade total, segundo o critério de notas para antúrio, estabelecido por Dias-Tagliacozzo \& Castro (2001b), sendo: Nota 4: aspecto geral excelente, plantas túrgidas, espata e espádice com brilho; Nota 3: aspecto geral bom, início da perda de turgescência e brilho da espata; Nota 2: aspecto geral regular, perda de turgescência, observada por meio da espata opaca e da mudança de coloração da haste floral, no local de inserção da espata; Nota 1: aspecto geral ruim, espata e espádice sem brilho, aspecto desbotado, haste com riscas de necrose; e Nota 0: descarte, espádice escura com ápice necrosado, espata murcha, haste amarela. Considerou-se como índice de durabilidade comercial, a média igual ou superior à nota 3 . A longevidade total compreendeu o período em dias, do momento da colheita até o descarte.

Os resultados foram submetidos à análise de variância e ao teste F, e, quando significativos, as médias foram comparadas pelo teste de Tukey, a 5\% de probabilidade.

\section{RESULTADOS E DISCUSSÃO}

Não se observou interação entre os cultivares e as soluções condicionantes, quanto ao critério de notas de inflorescências de antúrio, no quarto e oitavo dias após o condicionamento (Tabela 1).

Independentemente da solução condicionante, no décimo segundo dia após o condicionamento observou-se que os cultivares 'IAC Juréia' e 'IAC Eidibel' apresentaram as melhores notas $(3,98$ e 3,8), respectivamente, não diferindo entre si. Nas avaliações seguintes, o cultivar 'IAC Juréia' sobressaiu-se dos demais, sendo que, no décimo sexto dia, observou-se redução drástica na qualidade das inflorescências dos cultivares 'IAC Eidibel' e 'Apalai', com índice de durabilidade comercial abaixo do adequado (nota 3,0 ), com médias de 2,48 e 0,61 , respectivamente, sendo 'Apalai' o que perdeu mais rapidamente a qualidade, após esse período de condicionamento (Tabela 1).

Normalmente, a vida útil das hastes florais de antúrio está relacionada com a incapacidade de absorção de água (Reid \& Dodge, 2001), acompanhada de mudanças visíveis nas inflorescências, como a perda da textura, do brilho, da turgescência e a descoloração da espata (azulamento), o escurecimento e a necrose da espádice, o colapso da haste e a abscisão da espata e da espádice (Mujaffar \& Sankat, 2003), sendo estes sintomas observados nos cultivares em estudo, ao final do experimento.

A partir dos 28 dias após o condicionamento, constatou-se interação entre o cultivar 'Juréia' e as soluções condicionantes, sendo que a solução água + ácido cítrico apresentou melhores resultados, porém sem diferir daqueles da solução água + sacarose, e mantendo a qualidade até aos 40 dias após o condicionamento. Porém, esse resultado positivo não se refletiu na durabilidade comercial do cultivar 'Juréia', pois este manteve o índice de durabilidade comercial adequado somente até o vigésimo primeiro dia após o condicionamento. As longevidades dos cultivares 'IAC Juréia', 'IAC Eidibel' e 'Apalai' foram de 48, 36 e 28 dias, respectivamente (Tabela 1). 
Tabela 1. Qualidade de hastes florais de cultivares de antúrio, mantidos em diferentes soluções de condicionamento. APTA, Pariquera-Açú/SP, 2009

\begin{tabular}{|c|c|c|c|c|c|c|c|c|c|}
\hline \multicolumn{5}{|c|}{4 dias após o condicionamento } & \multicolumn{4}{|c|}{8 dias após o condicionamento } & \multirow{3}{*}{ Médias } \\
\hline \multirow[t]{2}{*}{ Cultivares } & \multicolumn{3}{|c|}{ Solução de condicionamento } & \multirow[t]{2}{*}{ Médias } & \multirow[t]{2}{*}{ Cultivares } & \multicolumn{3}{|c|}{ Solução de condicionamento } & \\
\hline & $\mathbf{A}$ & $\mathrm{A}+\mathrm{SC}$ & $\mathrm{A}+\mathrm{AC}$ & & & $\mathbf{A}$ & $\mathrm{A}+\mathrm{SC}$ & $\mathbf{A}+\mathbf{A C}$ & \\
\hline IAC Juréia & 4,00 & 4,00 & 4,00 & 4,00 & IAC Juréia & $4,00 \mathrm{a}$ & 4,00 & 4,00 & 4,00 \\
\hline IAC Eidibel & 4,00 & 4,00 & 4,00 & 4,00 & IAC Eidibel & $4,00 \mathrm{a}$ & 4,00 & 4,00 & 4,00 \\
\hline Apalai & 4,00 & 4,00 & 4,00 & 4,00 & Apalai & $3,90 \mathrm{~b} \mathrm{~B}$ & $4,00 \mathrm{~A}$ & $4,00 \mathrm{~A}$ & 3,95 \\
\hline \multirow[t]{2}{*}{ Médias } & 4,00 & 4,00 & 4,00 & & Médias & 3,95 & 4,00 & 4,00 & \\
\hline & \multicolumn{4}{|c|}{12 dias após o condicionamento } & \multicolumn{4}{|c|}{16 dias após o condicionamento } & \\
\hline \multirow[t]{2}{*}{ Cultivares } & \multicolumn{3}{|c|}{ Solução de condicionamento } & \multirow[t]{2}{*}{ Médias } & \multirow[t]{2}{*}{ Cultivares } & \multicolumn{3}{|c|}{ Solução de condicionamento } & Médias \\
\hline & A & $\mathrm{A}+\mathrm{SC}$ & $\mathbf{A}+\mathbf{A C}$ & & & & $\mathbf{A}$ & $\mathrm{A}+\mathrm{SC}$ & $\mathbf{A}+\mathbf{A C}$ \\
\hline IAC Juréia & $4,00 \mathrm{a}$ & 4,00 & 3,93 & $3,98 \mathrm{a}$ & IAC Juréia & $3,42 \mathrm{a}$ & $3,60 \mathrm{a}$ & $3,42 \mathrm{a}$ & $3,48 \mathrm{a}$ \\
\hline IAC Eidibel & $3,83 b$ & 3,85 & 3,75 & $3,80 \mathrm{ab}$ & IAC Eidibel & $2,17 b$ & $2,50 \mathrm{~b}$ & $2,75 \mathrm{a}$ & $2,48 b$ \\
\hline Apalai & $3,55 \mathrm{c}$ & 3,58 & 3,78 & $3,63 \mathrm{~b}$ & Apalai & $0,50 \mathrm{c}$ & $1,08 \mathrm{c}$ & $0,25 b$ & $0,61 \mathrm{c}$ \\
\hline Médias & 3,79 & 3,81 & 3,82 & & Médias & 2,03 & & 2,39 & 2,14 \\
\hline \multicolumn{5}{|c|}{20 dias após o condicionamento } & \multicolumn{4}{|c|}{24 dias após o condicionamento } & \\
\hline \multicolumn{4}{|c|}{ CultivaresSolução de condicionamento } & Médias & \multicolumn{4}{|c|}{ CultivaresSolução de condicionamento } & Médias \\
\hline & $\mathbf{A}$ & $\mathrm{A}+\mathrm{SC}$ & $\mathbf{A}+\mathbf{A C}$ & & & & $\mathbf{A}$ & $\mathrm{A}+\mathrm{SC}$ & $\mathrm{A}+\mathrm{AC}$ \\
\hline IAC Juréia & $2,95 \mathrm{a}$ & $3,25 \mathrm{a}$ & $3,28 \mathrm{a}$ & $3,16 \mathrm{a}$ & IAC Juréia & $2,18 \mathrm{a}$ & $2,58 \mathrm{a}$ & $2,73 \mathrm{a}$ & $2,49 \mathrm{a}$ \\
\hline IAC Eidibel & $1,40 \mathrm{~b}$ & $1,43 b$ & $1,08 b$ & $1,30 \mathrm{~b}$ & IAC Eidibel & $1,00 \mathrm{~b}$ & $1,25 b$ & $0,60 \mathrm{~b}$ & $0,95 b$ \\
\hline Apalai & $0,08 \mathrm{c}$ & $0,08 \mathrm{c}$ & $0,00 \mathrm{~b}$ & $0,05 \mathrm{c}$ & Apalai & $0,08 \mathrm{~b}$ & $0,00 \mathrm{c}$ & $0,00 \mathrm{~b}$ & $0,03 \mathrm{c}$ \\
\hline Médias & 1,48 & 1,58 & 1,45 & & Médias & 1,08 & 1,28 & 1,11 & \\
\hline \multicolumn{5}{|c|}{28 dias após o condicionamento } & \multicolumn{4}{|c|}{32 dias após o condicionamento } & \\
\hline \multicolumn{4}{|c|}{ CultivaresSolução de condicionamento } & Médias & Cultivares & olução de & ondicionan & ento & Médias \\
\hline & $\mathbf{A}$ & $\mathbf{A}+\mathbf{S C}$ & $\mathbf{A}+\mathbf{A C}$ & & A & $\mathrm{A}+\mathrm{SC}$ & $\mathrm{A}+\mathrm{AC}$ & & \\
\hline IAC Juréia & $1,33 \mathrm{a} \mathrm{B}$ & $1,78 \mathrm{a} A B$ & $2,25 \mathrm{a} \mathrm{A}$ & $1,78 \mathrm{a}$ & IAC Juréia & $1,08 \mathrm{a}$ B & $1,70 \mathrm{a} A B$ & $2,18 \mathrm{aA}$ & $1,65 \mathrm{a}$ \\
\hline IAC Eidibel & $0,33 b$ & $0,25 b$ & $0,25 b$ & $0,28 \mathrm{~b}$ & IAC Eidibel & $0,18 \mathrm{~b}$ & $0,15 b$ & $0,00 \mathrm{~b}$ & $0,11 \mathrm{~b}$ \\
\hline Apalai & $0,00 \mathrm{~b}$ & $0,00 \mathrm{~b}$ & $0,00 \mathrm{~b}$ & $0,00 \mathrm{~b}$ & Apalai & $0,00 \mathrm{~b}$ & $0,00 \mathrm{~b}$ & $0,00 \mathrm{~b}$ & $0,00 \mathrm{~b}$ \\
\hline Médias & 0,55 & 0,68 & 0,83 & & Médias & 0,42 & 0,62 & 0,73 & \\
\hline & 36 dias : & ós o condi & namento & & $40 \mathrm{~d}$ & ias após o & ndicionam & nto & \\
\hline CultivaresS & lução de & ondicionan & & Médias & Cultivares & olução de & ondicionan & ento & Médias \\
\hline & $\mathbf{A}$ & $\mathrm{A}+\mathrm{SC}$ & $\mathrm{A}+\mathrm{AC}$ & & $\mathbf{A}$ & $\mathrm{A}+\mathrm{SC}$ & $\mathrm{A}+\mathrm{AC}$ & & \\
\hline IAC Juréia & $0,73 \mathrm{a} \mathrm{B}$ & $1,25 \mathrm{a} A B$ & $1,68 \mathrm{a} \mathrm{A}$ & $1,22 \mathrm{a}$ & IAC Juréia & 0,40a B & $0,93 \mathrm{a} A$ & $1,35 \mathrm{a} \mathrm{A}$ & $0,89 \mathrm{a}$ \\
\hline IAC Eidibel & $0,08 \mathrm{a}$ & 0,00b & $0,00 \mathrm{~b}$ & $0,03 b$ & IAC Eidibel & $0,00 \mathrm{a}$ & $0,00 \mathrm{~b}$ & $0,00 \mathrm{~b}$ & $0,00 \mathrm{~b}$ \\
\hline Apalai & $0,00 \mathrm{a}$ & $0,00 \mathrm{~b}$ & $0,00 \mathrm{~b}$ & $0,00 \mathrm{~b}$ & Apalai & $0,00 \mathrm{a}$ & $0,00 \mathrm{~b}$ & $0,00 \mathrm{~b}$ & $0,00 \mathrm{~b}$ \\
\hline Médias & 0,27 & 0,42 & 0,56 & & Médias & $0,13 \mathrm{~B}$ & $0,31 \mathrm{AB}$ & $0,45 \mathrm{~A}$ & \\
\hline & 44 dias : & ós o condi & namento & & $48 \mathrm{~d}$ & ias após o & ndicionam & nto & \\
\hline Cultivares & Soluçã & de condici & mento & Médias & Cultivares & Soluçã & de condici & amento & Médias \\
\hline & A & $\mathrm{A}+\mathrm{SC}$ & $\mathrm{A}+\mathrm{AC}$ & & & $\mathrm{A}+\mathrm{SC}$ & $\mathrm{A}+\mathbf{A C}$ & & \\
\hline IAC Juréia & $0,23 \mathrm{a} B$ & $0,48 \mathrm{a}$ B & $1,00 \mathrm{a} A$ & $0,57 \mathrm{a}$ & IAC Juréia & $0,00 \mathrm{a} B$ & $0,18 \mathrm{a} B$ & $0,50 \mathrm{a} A$ & $0,23 \mathrm{a}$ \\
\hline IAC Eidibel & 0,00a & $0,00 \mathrm{~b}$ & $0,00 \mathrm{~b}$ & $0,00 \mathrm{~b}$ & IAC Eidibel & $0,00 \mathrm{a}$ & $0,00 \mathrm{~b}$ & $0,00 \mathrm{~b}$ & $0,00 \mathrm{~b}$ \\
\hline Apalai & $0,00 \mathrm{a}$ & $0,00 \mathrm{~b}$ & $0,00 \mathrm{~b}$ & $0,00 \mathrm{~b}$ & Apalai & $0,00 \mathrm{a}$ & $0,00 \mathrm{~b}$ & $0,00 \mathrm{~b}$ & $0,00 \mathrm{~b}$ \\
\hline Médias & $0,08 \mathrm{~B}$ & $0,16 \mathrm{AB}$ & A & & Médias & 0,00B & $0,06 \mathrm{AB}$ & $0,17 \mathrm{~A}$ & \\
\hline
\end{tabular}

Médias seguidas por letras distintas, minúscula na coluna (entre cultivares) e maiúsculas nas linhas (entre solução de condicionamento) diferem pelo Teste de Tukey a 5\% de probabilidade. A: água; A + SC: água + sacarose (5,0\%); A + AC: água + ácido cítrico $\left(200 \mathrm{mg} \mathrm{L}^{-1}\right)$. Escalas de notas: 4: aspecto geral excelente, plantas túrgidas, espata e espádice com brilho; 3: aspecto geral bom, início da perda de turgescência e brilho da espata; 2: aspecto geral regular, perda de turgescência observada pela espata opaca e mudança de coloração na haste floral no local de inserção da espata; 1: aspecto geral ruim, espata e espádice sem brilho, aspecto desbotado, haste com riscas de necrose; 0: descarte, espádice escura com ápice necrosado, espata murcha, haste amarela. 
Existem poucos estudos, em pós-colheita de antúrio, especialmente no Brasil, porém Castro et al. (1983) estudaram a conservação pós-colheita de antúrios, utilizando soluções com água, sacarose, hidroxiquinolina, sacarose + hidroxiquinolina e nitrato de prata, e observaram que o melhor tratamento foi obtido quando se utilizou somente a solução de sacarose a 8,0 \%, com uma longevidade de três semanas (21 dias).

Em relação à percentagem de ganho ou perda de massa após o condicionamento, no cultivar 'Apalai' observou-se diferenças entre as soluções condicionantes, após o período de condicionamento (24 horas), em que a solução água + ácido cítrico possibilitou maior ganho de massa, porém sem apresentar diferenças em relação à solução testemunha (Tabela 2). Constatou-se, também, que o cultivar 'IAC Eidibel' apresentou maior percentagem de ganho de massa após o condicionamento $(0,96 \%)$, independentemente da solução condicionante, porém sem apresentar diferenças em relação ao ganho do cultivar 'Apalai' $(0,73 \%)$.

Após quatro dias do condicionamento, não se observou efeito das soluções no cultivar 'Apalai', mas em 'IAC Juréia' e 'IAC Eidibel' foram observados efeitos positivos da solução água + sacarose, porém sem apresentar diferenças com os resultados da solução testemunha (água) no cultivar 'IAC Juréia' (Tabela 2).
Castro (1984) observou que a sacarose exógena manteve o volume de matéria seca e o nível de substratos respiratórios, especialmente nas pétalas de cravo e, consequentemente, promoveu maior longevidade. Doi \& Reid (1995) verificaram que para Limonium o açúcar translocado acumulou-se nas flores e folhas, aumentando a concentração osmótica e diminuindo o potencial hídrico, com melhoria na capacidade de absorção e favorecendo a manutenção da turgidez das pétalas.

Moraes et al. (1997) constataram maior vida de Liatris spicata, utilizando sacarose na concentração de $10 \%$, na forma de "pulsing”, por 20 horas. Gonzaga et al. (2001) observaram aumento na durabilidade de cravos, quando mantidos em soluções contendo sacarose a 4,0,\% e germicida. Dias-Tagliacozzo \& Castro (2001a) observaram aumento da longevidade de hastes florais de Phalaenopsis amabilis Lindl, utilizando a sacarose a 2,0 \% e 200 mg.L.-1 de ácido cítrico, durante 24 horas. Stancato \& Cesarino (2000) verificaram o prolongamento da vida de flores cortadas de Laelia purpurata, com a adição de 1,0\% de sacarose na solução preservativa.

Aos 8, 12, 16 e 20 dias após o condicionamento, observou-se que novamente o cultivar 'Apalai' apresentou menor perda de massa, quando condicionado na solução água + sacarose, porém, sem apresentar diferença em relação à

Tabela 2. Percentagem de ganho e perda de massa em hastes florais de cultivares de antúrio, mantidos em diferentes soluções de condicionamento. APTA, Pariquera-Açú/SP, 2009

\begin{tabular}{|c|c|c|c|c|c|c|c|c|c|}
\hline \multicolumn{3}{|c|}{0 dias após o condicionamento } & \multicolumn{6}{|c|}{4 dias após o condicionamento } & \multirow{3}{*}{ Médias } \\
\hline \multirow[t]{2}{*}{ Cultivares } & \multicolumn{3}{|c|}{ Solução de condicionamento } & \multirow[t]{2}{*}{ Médias } & \multirow{2}{*}{$\begin{array}{c}\text { Cultivares } \\
\text { A } \\
\end{array}$} & \multicolumn{3}{|c|}{ Solução de condicionamento } & \\
\hline & $\mathbf{A}$ & $\mathrm{A}+\mathrm{SC}$ & $\mathrm{A}+\mathrm{AC}$ & & & $\mathrm{A}+\mathrm{SC}$ & $\mathrm{A}+\mathrm{AC}$ & & \\
\hline IAC Juréia & 100,38 & 100,50 & 100,63 & $100,50 \mathrm{~b}$ & IAC Juréia & $99,65 b$ B & $100,60 \mathrm{a} A$ & $99,93 \mathrm{~b} \mathrm{~B}$ & $100,06 \mathrm{~b}$ \\
\hline IAC Eidibel & 101,00 & 100,93 & 100,95 & $100,96 a b$ & IAC Eidibel & $100,33 \mathrm{a} \mathrm{A}$ & $100,38 \mathrm{ab} A$ & $99,93 b$ B & $100,21 \mathrm{ab}$ \\
\hline Apalai & $100,58 \mathrm{AB}$ & $100,35 \mathrm{~B}$ & $101,25 \mathrm{~A}$ & $100,73 \mathrm{a}$ & Apalai & $100,30 \mathrm{a}$ & $100,18 b$ & $100,30 \mathrm{a}$ & $100,26 a$ \\
\hline Médias & 100,65 & 100,59 & 100,94 & & Médias & $100,09 \mathrm{~B}$ & $100,38 \mathrm{~A}$ & $100,05 \mathrm{~B}$ & \\
\hline \multicolumn{9}{|c|}{8 dias após o condicionamento } & \\
\hline \multirow[t]{2}{*}{ Cultivares } & \multicolumn{3}{|c|}{ Solução de condicionamento } & \multirow[t]{2}{*}{ Médias } & \multirow{2}{*}{$\begin{array}{c}\text { Cultivares } \\
\text { A } \\
\end{array}$} & \multicolumn{3}{|c|}{ Solução de condicionamento } & Médias \\
\hline & $\mathbf{A}$ & $\mathrm{A}+\mathrm{SC}$ & $\mathbf{A}+\mathbf{A C}$ & & & $\mathbf{A}+\mathbf{S C}$ & $\mathrm{A}+\mathrm{AC}$ & & \\
\hline IAC Juréia & $99,53 \mathrm{a}$ & 99,28 & 99,18 & $99,33 \mathrm{a}$ & IAC Juréia & $99,90 \mathrm{a}$ & $99,95 \mathrm{a}$ & $99,90 \mathrm{a}$ & $99,92 \mathrm{a}$ \\
\hline IAC Eidibel & $99,80 \mathrm{a}$ & 99,50 & 99,55 & $99,62 \mathrm{a}$ & IAC Eidibel & $99,20 \mathrm{a}$ & $98,93 \mathrm{ab}$ & $99,28 \mathrm{a}$ & $99,13 b$ \\
\hline Apalai & $97,60 \mathrm{~b} \mathrm{~B}$ & $99,05 \mathrm{~A}$ & $98,58 \mathrm{AB}$ & $98,41 b$ & Apalai & $96,43 b$ B & $98,10 \mathrm{~b} \mathrm{~A}$ & $96,58 \mathrm{~b} \mathrm{~B}$ & $97,03 \mathrm{c}$ \\
\hline Médias & 98,98 & 99,28 & 99,10 & & Médias & 98,51 & 98,99 & 98,58 & \\
\hline \multicolumn{9}{|c|}{16 dias após o condicionamento } & \\
\hline \multirow[t]{2}{*}{ Cultivares } & \multicolumn{3}{|c|}{ Solução de condicionamento } & Médias & Cultivares & \multicolumn{3}{|c|}{ Solução de condicionamento } & Médias \\
\hline & $\mathbf{A}$ & $\mathrm{A}+\mathrm{SC}$ & $\mathrm{A}+\mathrm{AC}$ & & $\mathbf{A}$ & $\mathrm{A}+\mathrm{SC}$ & $\mathbf{A}+\mathbf{A C}$ & & \\
\hline IAC Juréia & $99,73 a$ & $99,68 \mathrm{a}$ & $99,73 a$ & $99,71 \mathrm{a}$ & IAC Juréia & $99,45 \mathrm{a}$ & $99,33 \mathrm{a}$ & $99,98 \mathrm{a}$ & $99,58 \mathrm{a}$ \\
\hline IAC Eidibel & $96,93 \mathrm{a}$ & $96,80 \mathrm{ab}$ & $97,60 \mathrm{a}$ & $97,11 \mathrm{~b}$ & IAC Eidibel & $95,30 \mathrm{a}$ & $94,55 b$ & $95,08 b$ & $94,98 b$ \\
\hline Apalai & $92,33 \mathrm{~b} \mathrm{AB}$ & $94,88 \mathrm{~b} \mathrm{~A}$ & $91,95 \mathrm{~b} \mathrm{~B}$ & $93,05 \mathrm{c}$ & Apalai & $86,73 \mathrm{~b}$ B & $91,55 \mathrm{~b} \mathrm{~A}$ & $85,90 \mathrm{c} \mathrm{B}$ & $88,06 \mathrm{c}$ \\
\hline Médias & 96,33 & 97,12 & 96,43 & & Médias & 93,83 & 95,14 & 93,65 & \\
\hline
\end{tabular}

Médias seguidas por letras distintas, minúscula na coluna (entre cultivares) e maiúsculas nas linhas (entre solução de condicionamento) diferem pelo Teste de Tukey a $5 \%$ de probabilidade. A: água; A + SC: água + sacarose $(5,0 \%) ; \mathrm{A}+\mathrm{AC}$ : água + ácido cítrico (200 mg $\left.\mathrm{L}^{-1}\right)$. 
perda observada com a solução água + ácido cítrico, após oito dias de condicionamento, e com a solução testemunha, após 16 dias de condicionamento (Tabela 2). Essas perdas em massa fresca refletiram-se na queda drástica do índice de notas para o cultivar 'Apalai', com redução da durabilidade comercial e da longevidade total (Tabela 1).

Independentemente da solução condicionante, nas avaliações iniciais observou-se que o cultivar 'IAC Juréia' apresentou maiores perdas de massa, em relação às perdas dos demais cultivares ( 0 e 4 dias após o condicionamento). Depois desses períodos, houve maiores perdas de massa, principalmente para o cultivar 'Apalai', chegando, ao final das avaliações (20 dias após o condicionamento), com redução de $12,7 \%$ da massa inicial. Ao final da avaliação, observou-se que o cultivar 'IAC Juréia' apresentou menores perdas em massa $(0,4 \%)$, seguido do cultivar 'IAC Eidibel' (5,0\%). A causa mais comum do descarte das flores de corte é o murchamento, consequência de um estresse hídrico e não uma senescência natural, pois Elibox \& Umaharan (2010), que avaliaram a absorção de água em diferentes cultivares de antúrio, verificaram que cultivares com maior tempo de vida útil foram capazes de manter índices de absorção hídrica acima da média, por um período maior e, consequentemente, de retardar os sintomas causados pelo estresse hídrico.

Esses resultados mostram que, independentemente da solução condicionante, o fator cultivar é muito importante, na durabilidade comercial e na longevidade total, para inflorescências de antúrio, concordando com Nowak \& Rudnicki (1990), os quais relataram que a longevidade das flores é determinada por vários fatores pré e pós-colheita e, também, está relacionada com as características genéticas e anatômicas da espécie e dos cultivares.

Apesar de pesquisas recomendarem tratamentos póscolheita para aumentar a longevidade de inflorescências de antúrios, os tratamentos testados neste trabalho não resultaram em benefícios adicionais para os cultivares avaliados, pois, segundo Reid \& Dodge (2001), em alguns cultivares é possível obter longevidade total acima de três semanas após a colheita, se manuseados adequadamente, somente com a utilização de água deionizada. Essa informação está de acordo com os resultados observados neste trabalho, com a longevidade total dos cultivares 'Apalai', 'IAC Eidibel' e 'IAC Juréia' de 28, 36 e 48 dias, respectivamente, e as soluções de condicionamento não tendo aumentado a durabilidade comercial nem a longevidade total das inflorescências, ao final das avaliações.

\section{CONCLUSÕES}

A utilização de sacarose e de ácido cítrico, em solução de condicionamento por 24 horas, não causou aumento da durabilidade comercial e da longevidade total dos cultivares 'IAC Juréia', 'IAC Eidibel' e 'Apalai'.

O cultivar 'IAC Juréia' apresentou maior durabilidade comercial (21 dias) e longevidade total (48 dias), independentemente da solução de condicionamento.

\section{REFERÊNCIAS}

Castro ACR, Terao D, Carvalho ACPP \& Loges V (2012) Antúrio. Brasília, EMBRAPA. 163p.

Castro ACR, Resende LV, Guimarães WNR \& Loges V (2004) Uso de técnicas moleculares em estudo de diversidade genética em Anthurium. Revista Brasileira de Horticultura Ornamental, 10:6-9.

Castro CEF (1993) Helicônias como flores de corte: adequação de espécies e tecnologia pós-colheita. Tese de Doutorado. Escola Superior de Agricultura "Luiz de Queiroz", Piracicaba. 191p.

Castro CEF (1984) Tratamentos químicos pós-colheita e critérios de avaliação de qualidade de cravos (Dyanthus caryophyllus) cv. Scania Red Srin. Dissertação de Mestrado. Escola Superior de Agricultura "Luiz de Queiroz", Piracicaba. 139p.

Castro CEF, Matthes LAF \& Ferreira MA (1983) Conservação pós-colheita de antúrios. In: Congresso Brasileiro de Floricultura e Plantas Ornamentais, Rio de Janeiro. Anais, SBFPO. p.257263.

Dias-Tagliacozzo GM \& Castro CEF (2002) Fisiologia da póscolheita de espécies ornamentais. In: Wachowicz CM \& Carvalho RIN (Eds.) Fisiologia Vegetal: produção e pós-colheita. Curitiba, Champagnat. p.359-382.

Dias-Tagliacozzo GM \& Castro CEF (2001a) Prolongamento da longevidade pós-colheita de falenopse. In: $13^{\circ}$ Congresso Brasileiro de Floricultura e Plantas Ornamentais, São Paulo. Anais, Sociedade Brasileira de Floricultura e Plantas Ornamentais. p.27.

Dias-Tagliacozzo GM \& Castro CEF (2001b) Manutenção da qualidade pós-colheita de antúrio. In: $13^{\circ}$ Congresso Brasileiro de Floricultura e Plantas Ornamentais, São Paulo. Anais, Sociedade Brasileira de Floricultura e Plantas Ornamentais. p.30.

Doi M \& Reid MS (1995) Sucrose improves the postharvest life of cut flowers of hybrid Limonium. Hortscience, 30:1058-1060.

Elibox W \& Umaharan P (2010) Cultivar differences in the deterioration of vase-life in cut-flowers of Anthurium andraeanum is determined by mechanisms that regulate water uptake. Scientia Horticulturae, 124:102-108.

Gonzaga AR, Moreira LA, Lonardoni F \& Faria RT (2001) Longevidade pós-colheita de inflorescências de girassol afetada por nitrato de prata e sacarose. Revista Brasileira de Horticultura Ornamental, 7:73-77.

Govaerts R \& Frodin DG (2002) World checklist and bibliography of Araceae (and Acoraceae). Kew, Kew Publishing. 560p.

Halevy AH \& Mayak S (1981) Senescence and postharvest physiology of cut flowers. Part 2. In: Janick J (Ed.) Horticultural Reviews. Westport, AVI Publishing. p.59-143.

Kämpf AN (1984) Durabilidade das flores de corte (I). Lavoura Arrozeira, 37:14-15.

Leme JM (2008) Qualidade do antúrio 'IAC Eidibel' em cultivo hidropônico com fibra de coco. Tese de Doutorado. Universidade Estadual de Campinas, Campinas. 124p.

Lima JD \& Ferraz MV (2008) Cuidados na colheita e na póscolheita das flores tropicais. Revista Brasileira de Horticultura Ornamental, 14:29-34. 
Lopes LC \& Mantovani EC (1980) O cultivo de antúrios. Viçosa, Universidade Federal de Viçosa. 9p. (Boletim de Extensão, 22)

Lupa (2009) Levantamento censitário de unidades de produção agrícola do Estado de São Paulo. Disponível em: <http: ॥www.cati.sp.gov.br>. Acessado em: 5 de dezembro de 2011 .

Moraes PJ, Finger FL, Barbosa JG \& Silva DJH (1997) Efeito do "pulsing" com sacarose sobre o índice de sobrevivência de Chysanthemum leucanthemum L. Revista Brasileira de Horticultura Ornamental, 3:80-84.

Mujaffar S \& Sankat CK (2003) Effect of waxing on the water balance and keeping qualities of cut anthuriums. International Agrophysics, 17:77-84.

Nowak J \& Rudnicki RM (1990) Postharvest handling and storage of cut flowers, florist greens and potted plant. Portland, Timber Press. 210p.

Paull RE \& Goo T (1985) Ethylene and water stress in the senescence of cut anthurium flowers. Journal of the American Society for Horticultural Science, 110:84-88.
Reid MS \& Dodge L (2001) Anthurium: Recommendations for maintaining postharvest quality. Disponível em: <http:// rics.ucdavis.edu/postharvest2/Produce/ProduceFacts/Orn/ anthu.shtml>. Acessado em: 14 de dezembro de 2010.

Stancato GC \& Cesarino F (2000) Longevidade e durabilidade de flores de Laelia purpurata Lindl. (Orchidaceae). Revista Brasileira de Horticultura Ornamental, 6:69-74.

Tombolato AFC, Rivas EB, Coutinho LN, Bergmann EC, Imenes SL, Furlani PR, Castro CEF, Matthes LAF, Saes LA, Costa AMM, Tagliacozzo GMD \& Leme JM (2002) O cultivo de Antúrio: Produção Comercial. Campinas, Instituto Agronômico. 47p. (Boletim técnico, 194).

Tombolato AFC, Uzzo RP, Castro ACR, Sakai M \& Saes LA (2004) Recursos genéticos e melhoramento do antúrio (Anthurium andraeanum Linden) no IAC-APTA. Revista Brasileira de Horticultura Ornamental, 10:1-5.

Van Doorn WG \& Reid MS (1992) Role of ethylene in flower senescence of Gypsophila paniculata L. Postharvest Biology and Technology, 1:265-272. 